\title{
BMJ Open Identifying conducive contexts and working mechanisms of sedentary behaviour interventions in older adults: a realist review protocol as part of the 'Stand UP Seniors' project
}

\author{
Sofie Compernolle (D) , ${ }^{1}$ Delfien Van Dyck, ${ }^{1}$ Kenneth Vanhove, ${ }^{1}$ \\ Sebastien F M Chastin, ${ }^{2}$ Emelien Lauwerier, ${ }^{3,4}$ Greet Cardon ${ }^{5}$
}

To cite: Compernolle $\mathrm{S}$, Van Dyck D, Vanhove $\mathrm{K}$, et al. Identifying conducive contexts and working mechanisms of sedentary behaviour interventions in older adults: a realist review protocol as part of the 'Stand UP Seniors' project. BMJ Open 2021;11:e053942. doi:10.1136/ bmjopen-2021-053942

- Prepublication history and additional supplemental material for this paper are available online. To view these files, please visit the journal online (http://dx.doi.org/10.1136/ bmjopen-2021-053942).

Received 31 May 2021 Accepted 15 November 2021

Check for updates

(C) Author(s) (or their employer(s)) 2021. Re-use permitted under CC BY-NC. No commercial re-use. See rights and permissions. Published by BMJ.

For numbered affiliations see end of article.

Correspondence to

Sofie Compernolle;

sofie.compernolle@ugent.be

\section{ABSTRACT}

Introduction Lifestyle behaviours, including sedentary behaviour, have been listed as key modifiable factors to promote healthy ageing. Sedentary behaviour is ubiquitous in older adults and has a strong link with age-related functional declines and chronic health conditions. Although several interventions have been developed aimed at the reduction of sedentary behaviour in older adults, little in-depth information is available on how these complex interventions work in different contexts. Therefore, the aim of our study was to unpack the mechanisms of how existing interventions aimed at the reduction of older adults' sedentary behaviour work or fail to work in particular contexts in order to optimise the development and implementation of future sedentary behaviour interventions.

Methods and analysis A realist review will be conducted as a first part of the Stand UP Seniors (SUPS) project and will be structured as follows: (1) defining the scope of the review, (2) searching and appraising the evidence, (3) extracting data and synthesising the results, and (4) drawing conclusions and formulating recommendations. The result of this iterative process will be a final programme theory that can be used to identify which context triggers which mechanism, and in turn might elicit which outcome. The final programme theory will be used to inform the second and the third parts of the SUPS project, which are, respectively, the development and evaluation of a sedentary behaviour intervention in older adults.

Ethics and dissemination Ethical approval is not required for the review. Dissemination of the realist review results, including the final programme theory, will occur through peer-reviewed publications and presentations at relevant conferences. The peer-reviewed realist review will be prepared according to the Realist and Meta-narrative Evidence Synthesis: Evolving Standards publication standards for realist syntheses.

PROSPERO registration number CRD42021248795.

\section{INTRODUCTION}

Nowadays, people are living longer than ever before, thanks to improved living

\section{Strengths and limitations of this study}

- The explanatory focus of the realist methodology supports understanding the mechanisms through which, and the contexts in which, interventions aimed at the reduction of older adults' sedentary behaviour work or do not work.

- The iterative process used to build up the programme theory offers an innovative way to produce meaningful insights regarding effective implementation of sedentary behaviour interventions for older adults in different contexts.

- The formulation of a programme theory creates added value for stakeholders involved in the development and implementation of sedentary behaviour interventions for older adults.

- The limited availability of descriptive data regarding mechanisms of change and contextual elements forces the authors to make arguments based on their theoretical assumptions of what they believe are the mechanisms of action.

conditions and increasingly effective medical treatments. The average life expectancy in Western Europe is 79 years for men and 84 years for women, and is expected to continue rising in the next decades. ${ }^{1}$ Although longer life spans might appear to be good news at first sight, increased longevity is accompanied by functional decline and by the presence of long-term conditions, such as type 2 diabetes, heart disease, musculoskeletal disorders and cancer. ${ }^{2}$ A large-scale cross-sectional study with data from 1751841 Scottish people showed that $64.9 \%$ of adults aged above 65 years and $81.5 \%$ of adults aged above 85 years suffer from multimorbidity, meaning that they have two or more concomitant medical diagnoses. ${ }^{3}$ Functional decline and multimorbidity have been associated with several adverse effects, such as poor quality of life, ${ }^{4}$ increased levels 
of psychological distress ${ }^{5}$ and medical complications. ${ }^{6}$ These adverse effects strongly affect older adults' mental well-being ${ }^{7}$ and pose an economic burden on the healthcare system. ${ }^{8}$ A recent study conducted by The Organisation for Economic Co-operation and Development forecasted that the economic demands associated with long-term care will increase with about 7.7 percentage points of the gross domestic product between 2010 and $2060 .{ }^{9}$ Consequently, healthy ageing has become a global public health issue.

Besides promoting physical activity, reducing sedentary behaviour, defined as any waking behaviour characterised by an energy expenditure of 1.5 Metabolic Equivalent of Tasks (METS) or less when in a sitting, reclining or lying posture, ${ }^{10}$ has been pushed forward to promote healthy ageing. Although sedentary behaviour is ubiquitous in all age groups, older adults are the most sedentary segment of the population. ${ }^{11}$ Recent estimates show that older adults spend approximately $80 \%$ of their awake time in sedentary activities, which represents $8-12$ hours/day. ${ }^{12} 13$ Given the strong association between prolonged sedentary behaviour and the risk for negative health outcomes, such as frailty, physical disablement, type 2 diabetes, cardiovascular diseases and all-cause mortality, ${ }^{14-17}$ reducing sedentary behaviour seems promising. ${ }^{18}$

To date, a number of sedentary behaviour interventions have been specifically developed for older adults. Preliminary evidence regarding the effectiveness of sedentary behaviour interventions has been summarised in several reviews by answering the 'Does it work?' question. ${ }^{19-22}$ However, sedentary behaviour interventions are usually complex interventions, incorporating multiple behaviour change techniques, adopting different modes of delivery, including different stakeholders and agents required to engage in behaviour change, and being implemented in diverse settings. Due to this complex nature, it might be that particular intervention components work in one context, but not in another context, or that particular delivery modes are well suited for a specific subgroup of older adults but not for another. As such, the Does it work? question seems to be inadequate and insufficient. This is also reflected in the conclusion of existing systematic reviews, citing either a lack of clarity in the evidence base, uninterpretability of results or the need for better quality research to determine conclusions. Consequently, a new perspective is needed, focusing on what works for whom, under which circumstances and how. ${ }^{23}{ }^{24}$ This new perspective can be obtained using a so-called realist methodology. This methodology is particularly suitable for clarifying the process through which interventions may have impact by exploring the links between context, mechanism and outcome (CMO). Particularly, a realist review, as being an interpretative, theory-driven method, is ideal in synthesising existing evidence that uses crosscase comparison to understand and explain how and why different outcomes have been observed in a sample of studies on sedentary behaviour among older adults. ${ }^{25}$

\section{METHODS AND ANALYSIS \\ Project overview}

A realist review will be conducted as part of the Stand UP Seniors (SUPS) project. This project aims to gain a deep understanding of how, why and in which circumstances mobile health (mHealth) interventions aimed at the reduction of older adults' sedentary behaviour work by using a realist approach (see figure 1). The realist review will be the first step in the SUPS project. The result of

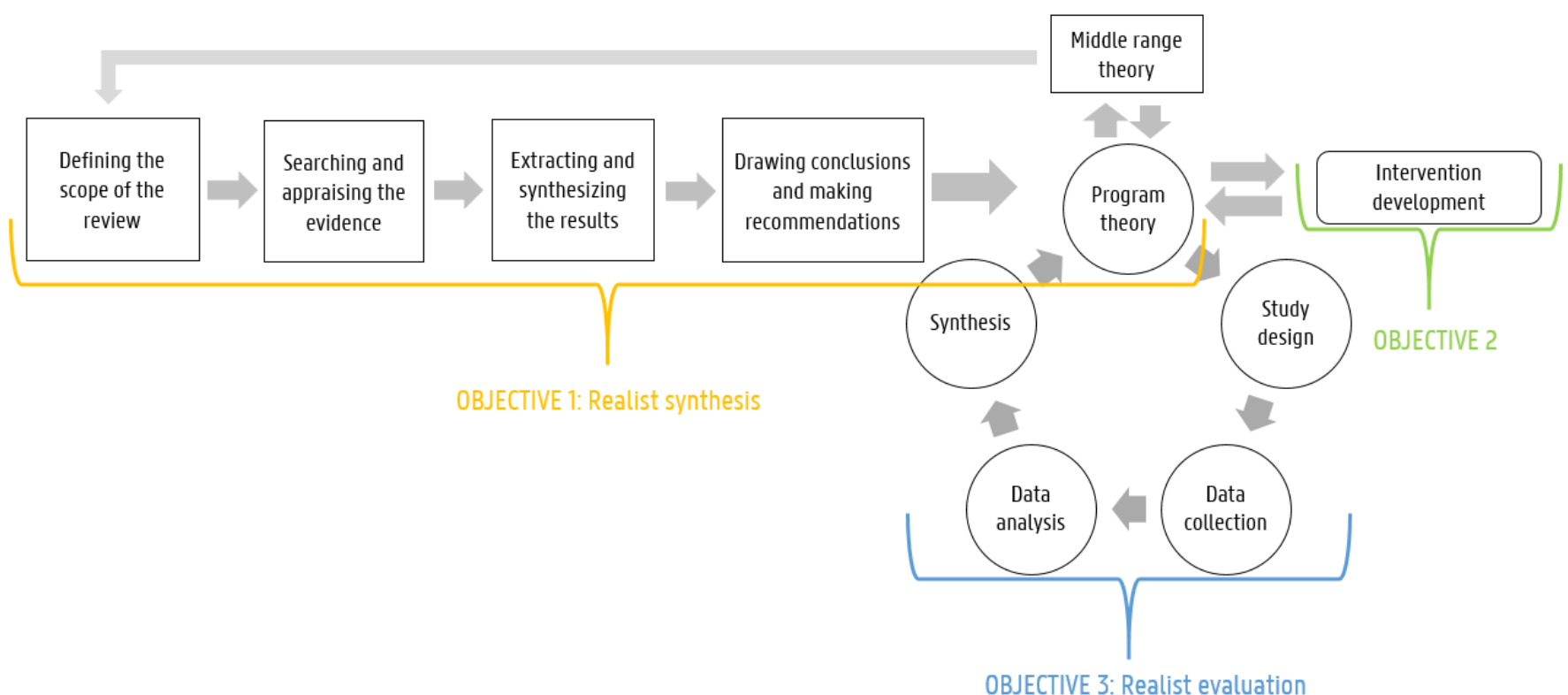

Figure 1 Overview of the Stand UP Seniors project. 
the realist review, that is, the programme theory, will be used to develop an mHealth intervention aimed at the reduction of older adults' sedentary behaviour during the second step. In the final step, the mHealth intervention will be assessed using a realist evaluation, and the results will be used to fine-tune the programme theory.

\section{Realist review}

A realist review differs from traditional meta-analyses and meta-syntheses, as the focus is explanatory (instead of evaluative), and the purpose is to understand how interventions work, with reference to particular contexts and settings (instead of demonstrating effectiveness). ${ }^{25}$ The synthesis process started in June 2021 and will follow four iterative stages as recommended by Pawson. ${ }^{26}$ First, the scope of the review was defined. Second, empirical evidence will be searched and appraised. Third, results will be extracted and synthesised into a final programme theory. This final programme theory can be used to identify contexts triggering mechanisms, leading to outcomes, which are referred to as the so-called CMO configurations, and to offer important insights for the development of new and the implementation of existing, sedentary behaviour interventions. The mechanisms of the CMO configurations will be subdivided, as described by Dalkin et $a l_{,}^{27}$ into resources (eg, intervention components/ modalities) and reasoning (eg, emotional and cognitive processes as response to the offered resources). Finally, conclusions will be drawn, and recommendations will be formulated based on the final programme theory (see figure 2). The realist review protocol is registered in the International Prospective Register of Systematic Reviews (registration number: CRD42021248795). The Preferred Reporting Items for Systematic Review and Meta-Analysis guidelines were used to structure the protocol (online supplemental file 1), ${ }^{28}$ and findings of the synthesis will be written up according to the Realist and Meta-narrative Evidence Synthesis: Evolving Standards (RAMESES) publication standards for realist syntheses. ${ }^{29}$

\section{Stage 1: define the scope of the review}

A realist review starts by clarifying the scope of the review and developing an initial programme theory, which provide the basis for the evidence synthesis. To clarify the scope of the review, KV and SC carefully read up and summarised the literature of sedentary behaviour interventions in older adults. SC provided an overview of the analytical themes derived from a recent thematic synthesis on older adults' perceptions of sedentary behaviour, ${ }^{30}$ and SFMC provided an overview of the most important findings of a recent Cochrane Review aimed at identifying the (cost-) effectiveness of sedentary behaviour interventions in older adults. ${ }^{22}$ Both the summary of the existing literature and the results of the thematic synthesis and Cochrane Review were presented to and discussed with the project group during the first project meeting. The project group includes (1) researchers with expertise in public health, health psychology and health promotion of older adults; (2) a staff member of the Flemish Institute of Healthy Living; and (3) the coordinator of the interdisciplinary consortium of Ghent University Research for Ageing Young. This interdisciplinary consortium bundles expertise on boosting healthy ageing from a wide variety of domains at Ghent University. The project group's input, in combination with the summary of the existing literature, was used to identify specific research questions and to refine the purpose of the review. The research questions are as follows: (1) what are the reasoning processes that drive the outcomes of sedentary behaviour interventions? (2) which intervention resources are needed to activate the reasoning processes that lead to sedentary behaviour reductions? (3) which contextual elements are conducive to trigger the reasoning processes that lead to sedentary behaviour reductions? Subsequently, SC and $\mathrm{KV}$ selected candidate middle-range theories by searching the literature and contacting experts, and SC drafted an initial programme theory. Although the terms 'middlerange theory' and 'programme theory' are increasingly used interchangeably, one could say that a middle-range theory is a generic theory about human psychology or sociology that introduce concepts to help explain how programmes work, whereas a programme theory is a hypothesis about how specific intervention resources (eg, information, advice, engagement and motivation) are expected to trigger specific mechanisms and outcomes

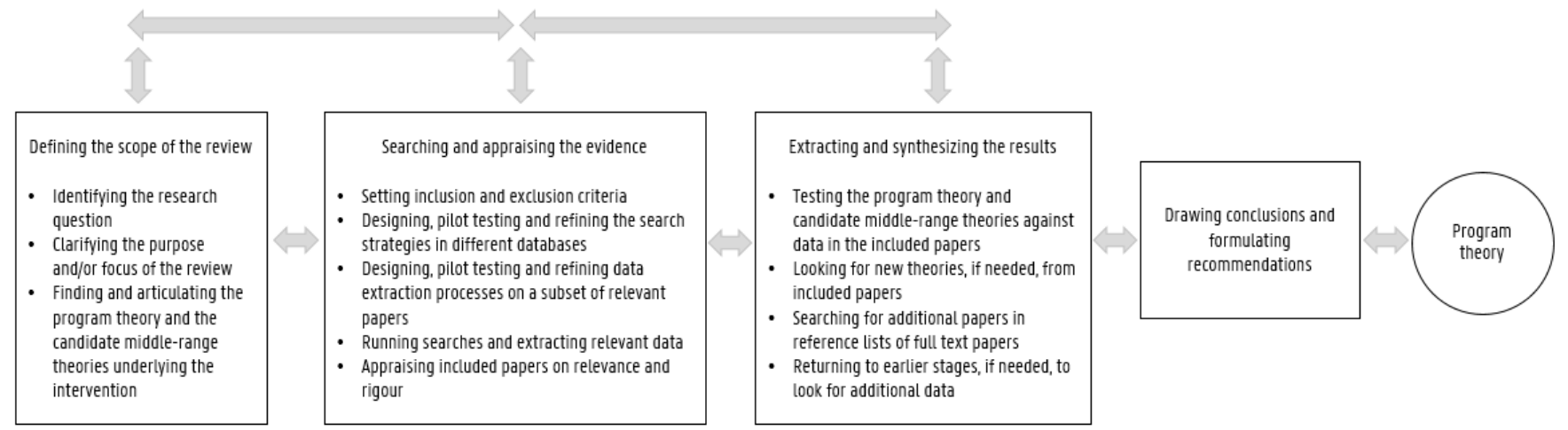

Figure 2 Realist synthesis stages adapted from Wong. ${ }^{34}$ 


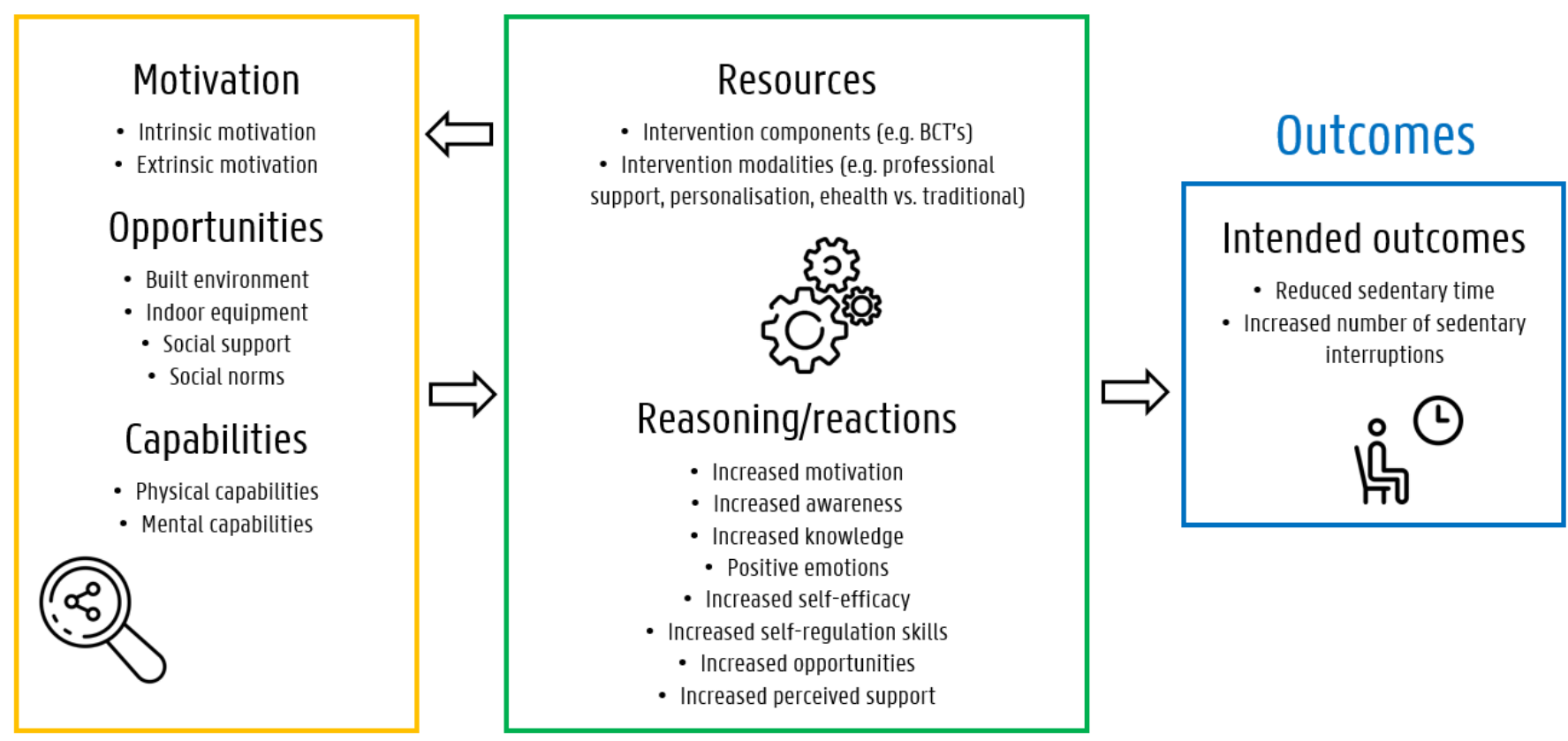

Figure 3 Initial programme theory.

in contexts. Middle-range theories are considered to be in between a grand universal theory and the programme theory. The middle-range theories used to formulate the programme theories were the dual-process theory of sedentary behaviour ${ }^{31}$ and the elaboration likelihood model of persuasion. ${ }^{32}$ Programme theories were refined and synthesised into one initial programme theory (see figure 3) using iterative discussions with the project team.

\section{Stage 2: search and appraise the evidence}

During the second stage of a realist review, existing empirical evidence relevant to the research questions and related to the initial programme theory will be purposively and iteratively searched and appraised. The search strategy was designed by SC and will be further refined in consultation with a medical librarian. The search strategy will include terms related to 'sedentary behaviour', 'older adults', and 'interventions', and will be entered in the following electronic databases: MEDLINE (PubMed interface), Embase (Embase.com interface), Web of Science and Scopus (see online supplemental file 2) for a first draft of the MEDLINE search strategy). The search will be limited to articles published in English since the beginning of 2000. This start date was chosen since most older studies used the construct sedentary behaviour as a synonym for physical inactivity. After running the search strategy in each of the databases, duplicates will be removed, and titles, abstracts and full texts of the retrieved articles will be independently reviewed by $\mathrm{KV}$ and SC during the article selection process. Studies will be eligible for inclusion during the selection process if they meet the following inclusion criteria: (1) conducted in older adults with a mean age of 65 years and above and (2) evaluating an intervention aimed at the reduction of sedentary behaviour. All research types (qualitative vs quantitative), study designs and settings will be included. When doubt regarding the inclusion of a study persists, a third reviewer will be consulted. Forward and backward reference checking of the included papers will be applied to identify additional relevant studies, and authors will be contacted to request for additional unpublished data. The Rayyan web application will be used to store search results and for the screening process. During the appraisal process, included papers will be judged on relevance and rigour. Papers will be considered relevant if they contain sufficient data to further refine (parts of) the initial programme theory. Irrelevant papers can still be excluded at this stage, and additional searches can be undertaken if the project team argues that insufficient data address certain parts of the programme theory. The additional searches will be planned by the project team and the medical librarian and performed by KV. Papers will be considered rigorous if the methods used to generate the relevant data are credible and trustworthy. Methodological quality as well as interpretations and claims of authors will be considered at this stage.

\section{Stage 3: extract data and synthesise the results}

Although data extraction belongs to the third stage of the realist review, data extraction will, in practice, often occur in parallel with the appraisal process. First, a standardised data extraction form will be created in Microsoft Excel to collect descriptive study and intervention information, including source characteristics (eg, author, 
year of publication and country of publication); study design; research questions; sample characteristics (eg, sample size, age and gender); intervention characteristics (eg, intervention content and mode of delivery); and study outcomes (eg, psychosocial outcomes and behavioural outcomes). Second, full texts of the selected papers will be uploaded in the qualitative analysis software programme, NVivo (QSR International, Melbourne, Australia). Sections containing relevant information to confirm, refute or refine the CMO configurations of the programme theory will be coded inductively (ie, datadriven, codes derived from the data itself), deductively (ie, theory-driven, codes originated from the predefined programme theory) and retroductively (ie, theoryguiding). Retroduction refers to the back-and-forth movements between known theories and the observed data to identify hidden causal mechanisms that might underlie emerging patterns. Retroduction is meant to overcome the deficiencies of induction and deduction. By coding the data, we will look for prominent demiregularities (ie, semipredictable occurring patterns) that might help us to better understand how sedentary behaviour interventions work in older adults. Both the data extraction and coding process will be undertaken by SC and KV independently. Inconsistencies or disagreements in the data extraction or coding process will be reflected on and discussed with the whole project team. Following the coding process, interpretive cross-case comparison will be performed by comparing successful sedentary behaviour intervention (components) (ie, those associated with positive outcomes) against non-successful intervention (components). By doing so, information on the contextual influence and the importance of the investigated mechanisms will be collected, allowing us to explain how and why observed outcomes have occurred, and to further refine the CMO configurations of the programme theory. In keeping with the iterative nature of the realist methodology, additional data might be searched, appraised and coded to further test the refined CMO configurations. A final consensus meeting will be convened with the project group, once synthesis of the literature is considered to be complete, to discuss, reflect and agree on the final programme theory.

\section{Stage 4: draw conclusions and formulate recommendations}

In the last stage, the CMO configurations of the final programme theory will be used to draw conclusions on how sedentary behaviour interventions work for whom, why and in what circumstances. Conclusions will be narratively reported and visually presented in a diagram. Conclusions will contain crucial information on the contexts, C, that are required for an (intermediate) outcome, $\mathrm{O}$, to happen, because of a certain mechanism, M. Once conclusions were reached, a stakeholder meeting will be held with researchers, practitioners and policy makers (1) to share the conclusions of the final programme theory and (2) to discuss recommendations for the development and implementation of future sedentary behaviour interventions. Involving stakeholders in this final phase is crucial to ensure that the recommendations of the realist review make sense to them and are neither lost nor ignored.

\section{Patient and public involvement}

Members of the public were not involved in the development of this protocol.

\section{Ethics and dissemination}

Ethical approval is not required for this review. Dissemination of the findings will be conducted through peerreviewed publications and presentations at relevant conferences. The peer-reviewed realist review will be prepared according to the RAMESES publication standards for realist syntheses ${ }^{29}$ and will be used to inform the next stages of the SUPS project. Concretely, the final programme theory will be used to develop prototypes of new sedentary behaviour interventions for older adults. Subsequently, microrandomised trials will be performed to evaluate (1) the average causal effects of the interventions on older adults' sedentary behaviour and (2) how these effects are moderated by participants' context. A microrandomised trial is an innovative experimental study design in health promotion research aimed to find out whether intervention A works better than intervention $\mathrm{B}$ or than no intervention. In contrast to standard randomised controlled trials, microrandomised trials are designed to provide data on the times and settings (ie, the context) when it is most effective to deliver each treatment option. ${ }^{33}$ Participants in microrandomised trials are sequentially randomised to different treatment options at each of many decision points at which treatment delivery might be effective (within subject design). In this way, researchers are allowed to assess the average causal effects of treatment on outcomes, as well as how these effects are moderated by participants' context (eg, time of the day, location, weather conditions or mood). By conducting the microrandomised trials, we aim to reach our ultimate goal of better understanding sedentary behaviour interventions in older adults.

\section{Author affiliations}

${ }^{1}$ Department of Movement and Sports Sciences, Ghent University, Gent, Belgium ${ }^{2}$ Institute of Applied Health Research, School of Health and Life Sciences, Glasgow Caledonian University, Glasgow, UK

${ }^{3}$ Department of Experimental Clinical and Healthy Psychology, Ghent University, Gent, Belgium

${ }^{4}$ Department of Public Health and Primary Care, Ghent University, Gent, Belgium

${ }^{5}$ Movement and Sports Sciences, Ghent University, Gent, Belgium

Contributors SC conceived the study with input from DVD, SFMC and GC. SC wrote the first draft of this article and will take the lead in the realist review. All authors provided feedback and suggestions to refine the manuscript and approved the final manuscript. SC was the guarantor of the review. SC and KV screened and appraised the articles, and extracted relevant data. They were supported by KV, DVD, SFMC, EL and GC during the review process.

Funding SC (FW020/PDJ/088) and KV (FW0.0PR.2021.0041.01) were supported by a postdoctoral fellowship and a project grant of the Research Foundation Flanders. The funders had no role in the study design, data collection and analysis, decision to publish or preparation of the manuscript. 
Competing interests None declared.

Patient consent for publication Not applicable.

Provenance and peer review Not commissioned; externally peer reviewed.

Supplemental material This content has been supplied by the author(s). It has not been vetted by BMJ Publishing Group Limited (BMJ) and may not have been peer-reviewed. Any opinions or recommendations discussed are solely those of the author(s) and are not endorsed by BMJ. BMJ disclaims all liability and responsibility arising from any reliance placed on the content. Where the content includes any translated material, BMJ does not warrant the accuracy and reliability of the translations (including but not limited to local regulations, clinical guidelines, terminology, drug names and drug dosages), and is not responsible for any error and/or omissions arising from translation and adaptation or otherwise.

Open access This is an open access article distributed in accordance with the Creative Commons Attribution Non Commercial (CC BY-NC 4.0) license, which permits others to distribute, remix, adapt, build upon this work non-commercially, and license their derivative works on different terms, provided the original work is properly cited, appropriate credit is given, any changes made indicated, and the use is non-commercial. See: http://creativecommons.org/licenses/by-nc/4.0/.

ORCID iD

Sofie Compernolle http://orcid.org/0000-0001-7742-2592

\section{REFERENCES}

1 Grundy EM, Murphy M. Population ageing in Europe. In: Oxford textbook of geriatric medicine. 11, 2017.

2 Banerjee S. Multimorbidity - older adults need health care that can count past one. Lancet 2015;385:587-9.

3 Barnett K, Mercer SW, Norbury M, et al. Epidemiology of multimorbidity and implications for health care, research, and medical education: a cross-sectional study. Lancet 2012;380:37-43.

4 Fortin M, Lapointe L, Hudon C, et al. Multimorbidity and quality of life in primary care: a systematic review. Health Qual Life Outcome 2004;2:51-2.

5 Fortin M, Bravo G, Hudon C, et al. Psychological distress and multimorbidity in primary care. Ann Fam Med 2006;4:417-22.

6 Fortin M, Soubhi H, Hudon C, et al. Multimorbidity's many challenges. BMJ 2007;334:1016-7.

7 Langan J, Mercer SW, Smith DJ. Multimorbidity and mental health: can psychiatry rise to the challenge? Br J Psychiatry 2013;202:391-3.

8 Picco L, Achilla E, Abdin E, et al. Economic burden of multimorbidity among older adults: impact on healthcare and societal costs. BMC Health Serv Res 2016;16:1-12.

9 De La Maisonneuve C, Martins JO. Public spending on health and long-term care 2013.

10 Tremblay MS, Aubert S, Barnes JD, et al. Sedentary behavior research network (SBRN) - terminology consensus project process and outcome. Int J Behav Nutr Phys Act 2017;14:75

11 Leask CF, Harvey JA, Skelton DA, et al. Exploring the context of sedentary behaviour in older adults (what, where, why, when and with whom). Eur Rev Aging Phys Act 2015;12:4.

12 Matthews CE, Chen KY, Freedson PS. Amount of time spent in sedentary behaviors in the United States, 2003-2004 2008;167:875-81.

13 Wilson JJ, McMullan I, Blackburn NE, et al. Associations of sedentary behavior bouts with community-dwelling older adults' physical function. Scand J Med Sci Sports 2021;31:153-62.
14 de Rezende LFM, Rey-López JP, Matsudo VKR, et al. Sedentary behavior and health outcomes among older adults: a systematic review. BMC Public Health 2014;14:333.

15 Copeland JL, Ashe MC, Biddle SJ, et al. Sedentary time in older adults: a critical review of measurement, associations with health, and interventions. Br J Sports Med 2017;51:1539.

16 Rosenberg DE, Bellettiere J, Gardiner PA. Independent associations between sedentary behaviors and mental, cognitive, physical, and functional health among older adults in retirement communities 2015;71:78-83

17 Santos DA, Silva AM, Baptista F, et al. Sedentary behavior and physical activity are independently related to functional fitness in older adults. Exp Gerontol 2012;47:908-12.

18 DiPietro L, Al-Ansari SS, Biddle SJH, et al. Advancing the global physical activity agenda: recommendations for future research by the 2020 who physical activity and sedentary behavior guidelines development group. Int J Behav Nutr Phys Act 2020;17:1-11.

19 Chase J-AD, Otmanowski J, Rowland S, et al. A systematic review and meta-analysis of interventions to reduce sedentary behavior among older adults. Trans/ Behav Med 2020;10:1078-85.

20 Stockwell S, Schofield P, Fisher A, et al. Digital behavior change interventions to promote physical activity and/or reduce sedentary behavior in older adults: a systematic review and meta-analysis. Exp Gerontol 2019;120:68-87.

21 Aunger JA, Doody P, Greig CA. Interventions targeting sedentary behavior in non-working older adults: a systematic review. Maturitas 2018;116:89-99.

22 Chastin S, Gardiner PA, Ashe MC, et al. Interventions for reducing sedentary behaviour in community-dwelling older adults. Cochrane Database Syst Rev 2017;2017.

23 Jagosh J. Realist synthesis for public health: building an ontologically deep understanding of how programs work, for whom, and in which contexts. Annu Rev Public Health 2019;40:361-72.

24 Pawson R, Tilley N. An introduction to scientific realist evaluation. In: Evaluation for the 21st century: a handbook, 1997: 405-18.

25 Pawson R, Greenhalgh T, Harvey G, et al. Realist review-a new method of systematic review designed for complex policy interventions. J Health Serv Res Policy 2005;10:21-34.

26 Pawson R. Tilley NJEftscAh: an introduction to scientific realist evaluation 1997:405-18.

27 Dalkin SM, Greenhalgh J, Jones D, et al. What's in a mechanism? Development of a key concept in realist evaluation. Implement Sci 2015;10:1-7.

28 Moher D, Shamseer L, Clarke M, et al. Preferred reporting items for systematic review and meta-analysis protocols (PRISMA-P) 2015 statement. Syst Rev 2015;4:1-9.

29 Wong G, Greenhalgh T, Westhorp G, et al. RAMESES publication standards: realist syntheses. BMC Med 2013;11:1-14.

30 Compernolle S, De Cocker K, Cardon G. Older adults' perceptions of sedentary behavior: a systematic review and thematic synthesis of qualitative studies. Gerontologist2019.

31 Maher JP, Conroy DE. A dual-process model of older adults' sedentary behavior. Health Psychol 2016;35:262-72.

32 Petty RE, Cacioppo JT. The elaboration likelihood model of persuasion. In: Communication and persuasion. Springer, 1986: 1-24.

33 Seewald NJ, Smith SN, Lee AJ, et al. Practical considerations for data collection and management in mobile health micro-randomized trials. Stat Biosci 2019;11:355-70.

34 Sqrctra CJW, Wong GJ. The internet in medical education: a worked example of a realist review 2012:83-112. 